\section{Complete Genome Sequence Data for the Grapevine Crown Gall-Inhibiting Bacteria Allorhizobium vitis F2/5}

\author{
Hangwei Xi, ${ }^{1}$ Josh Grist, ${ }^{1}$ Maarten Ryder, ${ }^{2}$ and lain R. Searle ${ }^{1, \dagger}$ \\ ${ }^{1}$ School of Biological Sciences, The University of Adelaide, Adelaide, 5005, Australia \\ ${ }^{2}$ School of Agriculture, Food and Wine, The University of Adelaide, Adelaide, 5064, Australia
}

\section{Genome Announcement}

Crown gall disease in grapevine is caused by pathogenic strains of Allorhizobium vitis. A. vitis strain F2/5 is a nonpathogenic biocontrol agent that was previously shown to act as a biological control agent to crown gall disease and was first isolated from South Africa. Here, we present the complete assembled genome, which is $5.94 \mathrm{Mb}$ in length with 5,414 predicted protein-coding sequences and has two circular chromosomes and five plasmids. The genome sequence has no detectable T-DNA border sequences and is missing key virulence genes, which is consistent with the bacteria being nonpathogenic. The F2/5 genome sequence could contribute to understanding the molecular basis underlying the biocontrol activity.

Grapevine crown gall caused by Agrobacterium vitis (now reclassified to the genus Allorhizobium [Mousavi et al. 2014]) is a serious chronic disease that can cause decreased production or early grapevine death (Gillings and Ophel-Keller 1995). Grape crown gall can be partially managed through varietal selections, cultural practices and can be suppressed to varying degrees in greenhouse experiments by biocontrol agents (Zheng and Burr 2016). Biocontrol agents include $A$. vitis strains ARK1, E26, VAR03-1, and F2/5 (Kawaguchi 2013; Kawaguchi et al. 2008; Liang 1990; Staphorst et al. 1985). Strain F2/5 is nontumorigenicm and the antibiosis conferred against pathogenic $A$. vitis strains is thought to occur by inhibiting transformation of the grape tissue by the pathogen as opposed to inhibiting attachment of the pathogen to wounded cells or growth of the pathogen in the wounded tissue (Kaewnum et al. 2013). The antibiosis or grape tumor inhibition (GTI) conferred by F2/5 requires living cells, whereas heat-killed or sonicated-lysed cell preparations lose GTI ability (Burr et al. 1997). A number of genes were identified that are required for GTI, including clpA ( $F$-avi2537), alpP1 (F-avi1696), aviR (F-avi4374) (Zheng and Burr 2016). To date, no genome sequences of the potential biocontrol strains ARK1, E26, and F2/5 have been published, but the genome sequence for VAR03-1 has been recently published (Noutoshi et al. 2020).

A single colony of F2/5 (Burr collection, Cornell University, Ithaca, NY, U.S.A.) was inoculated into $6 \mathrm{ml}$ of yeast mannitol broth and was grown overnight at $28^{\circ} \mathrm{C}$. Genomic DNA purification was performed, using a PowerSoil kit (Qiagen). After shearing the purified DNA to 10 to $15 \mathrm{~Kb}$ with a 26-gauge needle, a Blue Pippin (Sage Science) was used to select fragments between 10 and $25 \mathrm{~Kb}$. For PacBio library preparation and sequencing, DNA was processed with the SMRTbell template prep kit 1.0 (PacBio) and was sequenced on a PacBio Sequel instrument. A total of $2.25 \mathrm{~Gb}$ of sequencing data with a read $\mathrm{N}_{50}$ of $6.20 \mathrm{~Kb}$ was obtained, representing approximately $374 \times$ coverage.

${ }^{\dagger}$ Corresponding author: I. R. Searle; iain.searle@ adelaide.edu.au

The author(s) declare no conflict of interest.

Accepted for publication 27 October 2021.

\section{Funding}

This work was supported by funding from the University of Adelaide (Australia) by a postgraduate scholarship awarded to $\mathrm{H}$. Xi and research funds awarded to $I$. $R$. Searle.

\section{Keywords}

Agrobacterium vitis, Allorhizobium vitis, biological control, grapevine crown gall 
Table 1. Genomic features of the Agrobacterium vitis F2/5 genome ${ }^{a}$

\begin{tabular}{lllllllll} 
Name & Contig 1 & Contig 2 & & Contig 3 & Contig 4 & \multicolumn{1}{c}{ Contig 5 } & Contig 6 & Contig 7 \\
Feature & Chr. 1 & Chr. 2 & Partial virulence region & Nopaline & Nopaline Acc operon & ttuC & $\ldots$ \\
Size (bp) & $3,663,811$ & $1,185,416$ & 98,206 & 150,180 & 200,742 & 204,449 & 436,826 \\
Circular & Yes & Yes & Yes & 97 & Yes & Yes & Yes & Yes \\
Coding sequence & 3,374 & 991 & 0 & 139 & 197 & 220 & 396 \\
Ribosomal RNAs & 9 & 3 & 0 & 0 & 0 & 0 & 0 \\
Transfer RNAs & 52 & 4 & 0 & 0 & 0 & 0 \\
\hline
\end{tabular}

${ }^{\mathrm{a}}$ Chr. = chromosome; Acc = agrocinopine uptake and catabolism.

A genome assembly was built from the PacBio sequence reads, using Flye v2.7 (Kolmogorov et al. 2019). The assembly consisted initially of eight contigs with an $\mathrm{N}_{50}$ value of $3.07 \mathrm{Mb}$. The PacBio reads were mapped back to the draft genome assembly, using Minimap2 v2.22 (Li 2018), and the mapping rate was 98.6\%. BUSCO v5.2.2 (Simão et al. 2015) identified $97.0 \%$ complete genes in the assembly of 1,937 markers in the database rhizobium-agrobacterium_group_odb10, suggesting high integrity and completeness of the assembly. LASTZ (Harris 2007) was used to identify the similarity of each contig with closely related bacterial species, using the default parameters (step length $=20$, seed pattern $=12$ of 19 , HSP threshold score $=3,000$ [Geneious Prime plugin LASTZ v1.02.00]). Two contigs were very similar to $A$. vitis $S 4$ chromosome 1 , were contiguous, and were manually merged to form contig 1 . We showed contig 1 was contiguous in F2/5 by mapping the raw PacBio reads with Minimap2 (Geneious Prime v2021.2.2) and identified more than 50 reads spanning the junction. Contig 2 was very similar (average nucleotide identity $[\mathrm{ANI}]>95 \%$ ) to A. vitis S4 (GenBank accession GCA_000016285.1) chromosome 2. Accordingly, contig 1 was named chromosome 1 and contig 2 was named chromosome 2. ANI were calculated by using FastANI (v1.32) (Jain et al. 2018). All seven contigs were demonstrated as circular sequences by manually linking $10 \mathrm{~Kb}$ of the $3^{\prime}$ and $5^{\prime}$ ends and identifying spanning sequence reads, using Minimap2. Genome annotation was performed by using the DDBJ fast annotation and submission tool (DFAST) (Tanizawa et al. 2016).

The final, high-quality genome of seven contigs has a total length of $5.94 \mathrm{Mb}$, an $\mathrm{N}_{50}$ value of $3.66 \mathrm{Mb}$, and GC content of $57.6 \%$. DFAST identified 5,414 protein-coding sequences, 56 transfer RNAs and 12 ribosomal RNAs (Table 1). F2/5 is nonpathogenic on grapevine, and contig 3 harbors a partial virulence region with nine virulence-related genes (VirA, VirB8, VirB11, VirC1, VirC2, VirD1, VirD2, VirD4, and VirG). In contrast, pathogenic Agrobacterium tumefaciens $\mathrm{C} 58$ has 27 virulence genes on its Ti plasmid (Wood et al. 2001). Contigs 4 and 5 have genes for nopaline uptake and assimiliation. In addition, contig 5 has an agrocinopine uptake and catabolism operon. Contig 6 has a gene homologous to ttuC for tartrate utilization, typical of $A$. vitis (Xi et al. 2021). We searched the genome sequence for the consensus T-DNA border sequence NNTGGCAGGATATATNNNNNTGTAWN, and no identical sequence was found, which is consistent with the bacteria being nonpathogenic.

\section{Data Availability}

The complete genome sequence and associated data for $A$. vitis $\mathrm{F} 2 / 5$ were deposited under GenBank accession numbers CP081837 through CP081843, BioProject accession number PRJNA755483, Short Read Archive accession number SRR15522074, and BioSample accession number SAMN20826601.

\section{Literature Cited}

Burr, T. J., Reid, C. L., Tagliati, E., Bazzi, C., and Süle, S. 1997. Biological control of grape crown gall by strain $12 / 5$ is not associated with agrocin production or competition for attachment sites on grape cells. Phytopathology 87: 706-711.

Gillings, M., and Ophel-Keller, K. 1995. Comparison of strains of Agrobacterium vitis from grapevine source areas in Australia. Australas. Plant Pathol. 24: 29-37.
Harris, R. S. 2007. Improved pairwise alignment of genomic DNA. Ph.D. thesis. The Pennsylvania State University, State College, PA, U.S.A.

Jain, C., Rodriguez-R, L. M., Phillippy, A. M., Konstantinidis, K. T., and Aluru, S. 2018. High throughput ANI analysis of $90 \mathrm{~K}$ prokaryotic genomes reveals clear species boundaries. Nat. Commun. 9:5114.

Kaewnum, S., Zheng, D., Reid, C. L., Johnson, K. L., Gee, J. C., and Burr, T. J. 2013. A host-specific biological control of grape crown gall by Agrobacterium vitis strain F2/5: Its regulation and population dynamics. Phytopathology 103:427-435. 
Kawaguchi, A. 2013. Biological control of crown gall on grapevine and root colonization by nonpathogenic Rhizobium vitis strain ARK-1. Microbes Environ. 28:306-311.

Kawaguchi, A., Inoue, K., and Ichinose, Y. 2008. Biological control of crown gall of grapevine, rose, and tomato by nonpathogenic Agrobacterium vitis strain VAR03-1. Phytopathology 98:1218-1225.

Kolmogorov, M., Yuan, J., Lin, Y., and Pevzner, P. A. 2019. Assembly of long, error-prone reads using repeat graphs. Nat. Biotechnol. 37:540-546.

Li, H. 2018. Minimap2: Pairwise alignment for nucleotide sequences. Bioinformatics $34: 3094-3100$.

Liang, Y. 1990. A biotype 3 strain of Agrobacterium radiobacter inhibits crown gall formation on grapevine. Acta Microbiol. Sin. 30:165-171.

Mousavi, S. A., Österman, J., Wahlberg, N., Nesme, X., Lavire, C., Vial, L., Paulin, L., de Lajudie, P., and Lindström, K. 2014. Phylogeny of the Rhizobium-Allorhizobium-Agrobacterium clade supports the delineation of Neorhizobium gen. nov. Syst. Appl. Microbiol. 37:208-215.

Noutoshi, Y., Toyoda, A., Ishii, T., Saito, K., Watanabe, M., and Kawaguchi, A. 2020. Complete genome sequence data of nonpathogenic strain Rhizobium vitis VAR03-1, a biological control agent for grapevine crown gall disease. Mol. Plant-Microbe Interact. 33:1451-1453.
Simão, F. A., Waterhouse, R. M., loannidis, P., Kriventseva, E. V., and Zdobnov, E. M. 2015. BUSCO: Assessing genome assembly and annotation completeness with single-copy orthologs. Bioinformatics 31:3210-3212.

Staphorst, J. L., Van Zyl, F. G. H., Strijdom, B. W., and Groenewold, Z. E. 1985. Agrocin-producing pathogenic and nonpathogenic biotype-3 strains of Agrobacterium tumefaciens active against biotype-3 pathogens. Curr. Microbiol. 12:45-52.

Tanizawa, Y., Fujisawa, T., Kaminuma, E., Nakamura, Y., and Arita, M. 2016. DFAST and DAGA: Web-based integrated genome annotation tools and resources. Biosci. Microbiota Food Health 35:173-184.

Wood, D. W., Setubal, J. C., Kaul, R., Monks, D. E., Kitajima, J. P., Okura, V. K., Zhou, Y., Chen, L., Wood, G. E., Almeida, N. F. 2001. The genome of the natural genetic engineer Agrobacterium tumefaciens C58. Science 294:2317-2323.

Xi, H., Ryder, M., and Searle, I. R. 2021. Near-complete genome assembly of the grapevine crown gall pathogen Allorhizobium vitis strain K377. Microbiol. Resour. Announc. 10:e0135920.

Zheng, D., and Burr, T. J. 2016. Inhibition of grape crown gall by Agrobacterium vitis F2/5 requires two nonribosomal peptide synthetases and one polyketide synthase. Mol. Plant-Microbe Interact. 29:109-118. 